\title{
Acute toxicity variation of hydroxyl benzophenone UV filters during photoinduction-chlorination disinfection processes
}

\author{
Qi Yu, Dongbin Wei*, Wei Liu, Yuguo Du
}

1. State Key Laboratory of Environmental Chemistry and Ecotoxicology, Research Center for Eco-Environmental Sciences, Chinese Academy of Sciences, Beijing 100085, China. E-mail: yuqi6165@126.com

2. University of Chinese Academy of Sciences, Beijing 100049, China

\section{A R T I C L E I N F O}

Article history:

Received 4 March 2016

Revised 29 April 2016

Accepted 9 May 2016

Available online 30 June 2016

Keywords:

Benzophenones

Photo induction

Chlorination

Disinfection

Toxicity

\begin{abstract}
A B S T R A C T
Benzophenones (BPs), a group of widely used UV filters, exert multiple, significant toxicity effects. The 11 BPs were selected as target compounds, and the photobacterium acute toxicity test and an index for acute toxicity formation potential (ATFP) were used to evaluate the toxicity variation of BPs before and after a photoinduction-chlorination disinfection process. Orthogonal experiments were performed at different $\mathrm{pH}$ values and chlorine dosages. The characteristics of ATFP values for 11 BPs after a photoinduction-chlorination process can be summarized as follows: (1) The ATFPs decreased as the hydroxyl group number increased in BPs molecules. (2) For those BPs with the same hydroxyl group number, the ATFPs were higher when the hydroxyl groups were located at the 3- or 4-position than those at the 2-position; the BPs with hydroxyl groups distributed on two benzene rings had higher ATFPs than those on one ring. (3) Introducing a methoxyl group and sulfonic acid group into BP molecules increased the ATFP values. (4) The ATFPs were pH-dependent, the values of which were lowest at the neutral condition and highest at the acid condition. (5) The ATFPs increased and then decreased as the chlorine dosage increased. The results can be used as a reference to scientifically evaluate the environmental fate and potential risk of BPs in photoinductionchlorination disinfection processes.

(C) 2016 The Research Center for Eco-Environmental Sciences, Chinese Academy of Sciences. Published by Elsevier B.V.
\end{abstract}

\section{Introduction}

Over the past several decades, the depletion of the ozone layer has become increasingly serious and has resulted in more ultraviolet (UV) irradiation reaching Earth. As a result, the health threats from UV exposure, which include numerous skin diseases such as sunburns, photo-aging and even skin cancer, have raised wide public concerns (Pathak, 1987). Benzophenone (BP) chemicals are a group of widely used UV filters in sunscreens and cosmetics that protect from ultraviolet ray radiation due to their broad UV absorption spectrum (Roelandts et al., 1983). BPs are also used as a flavor ingredient, fragrance enhancer and additive in plastics, coatings, cleaning products and pharmaceuticals (Careghini et al., 2015). The maximum authorized concentration in cosmetics of BP-3 (2-hydroxy-4-methoxy-BP) is $10 \%$ in the $\mathrm{EU}$, that of $\mathrm{BP}-8$ $\left(2,2^{\prime}\right.$-dihydroxy-4-methoxy-BP) is $3 \%$ in Korea and that of BP-4 (2-hydroxy-4-methoxy-5-sulfonic acid-BP) is $10 \%$ in Japan (EEC, 1983; Jeon et al., 2006; MHLW, 2000). The production of BPs exceeded 453 tons in the USA and 10,000 tons in the European Union by 2003 (NTP, 2006). A large amount of BP residues and metabolites continuously enter the environment via recreational water activities or wastewater treatment plants (Gago-Ferrero et al., 2013).

\footnotetext{
* Corresponding author. E-mail: weidb@rcees.ac.cn (Dongbin Wei).
} 
Consequently, many BPs have been detected from multiple environmental matrices and biota, such as water (Almeida et al., 2013), sediment (Zhang et al., 2011), indoor dust (Wang et al., 2013) and fish (Fent et al., 2010). The residual concentrations of BP-4 have been found to be in the hundreds of ng/L in rivers and sea water and in the range of 237-1481 ng/L in wastewater (Rodil et al., 2008). The BP-3 level has been found to reach up to $238 \mathrm{ng} / \mathrm{L}$ in human blood (Janjua et al., 2008), and the concentration of BP-1 (2,4-dihydroxy-BP) has been found to range from 1 to $14.6 \mathrm{ng} / \mathrm{L}$ in human urine (Asimakopoulos et al., 2014a, 2014b). Many reports have revealed that BPs have multiple adverse biological effects. Among them, the estrogenic and anti-estrogenic activities of BPs are of great concerns. BP-2 (2,2',4,4'-tetrahydroxy-BP) can act as estrogenic disruptors to fish, leading to adverse effects on egg production and gonadal development (Weisbrod et al., 2007). BP-4 interfered with sex hormones and can alter genes transcription, including steroidogenesis, and hormonal pathways in zebrafish (Nancy et al., 2012). BP-2 can induce male Pimephales promelas to generate vitellogenin and weaken secondary sex characteristics (Weisbrod et al., 2007). Regarding humans, BP-1 has been associated with endometriosis, and BP-3 was found to stimulate the proliferation of the breast cancer cell line MCF-7 (Kunisue et al., 2012; Schlumpf et al., 2001). In particular, a current epidemiological survey showed that BP-2 and 4-HBP (4-hydroxy-BP) were associated with a $31 \%$ and $26 \%$ reduction in fecundity, respectively (Louis et al., 2014). Therefore, BPs have been defined as "chemicals suspected of having endocrine disrupting effects" by the Japanese Ministry of Environment (NIES, 1998). In addition, BPs also exhibit genotoxicity and acute toxicity. The 2,3,4-trihydroxy-BP was found to have a genotoxic effect on L5178Y cells (Jeon et al., 2007). BP-2 showed positive genotoxicity on Salmonella thyphimurium at $81.97 \mathrm{mg} / \mathrm{L}$ (Zhao et al., 2013). BPs exerted acute toxicity, where the EC50s of BP-8 were 10.48 and $3.55 \mathrm{mg} / \mathrm{L}$ on Photobacterium phosphoreum and Daphnia, respectively (Liu et al., 2015).

Disinfection with free available chlorine $\left(\mathrm{FAC}, \mathrm{HOCl} / \mathrm{OCl}^{-}\right)$is widely used to kill pathogens in water treatment processes. Since the 1970s, increasing amounts of carcinogenic by-products have been found in chlorination disinfection processes, such as trihalomethanes, haloacetate acids, haloacetonitriles and $\mathrm{N}$ nitrosodimethylamine (NDMA) (Richardson et al., 2007). To reduce the toxic by-product formation, new preferable disinfection methods have been explored. For example, in a UV-chlorination combined disinfection system, the formation of chloroform was found to be 12-fold less than that in a single chlorination disinfection system (Yang et al., 2011). Moreover, illumination, particularly ultraviolet, can effectively kill certain anti-chlorination disinfection protozoa, such as Cryptosporidium (Liu, 2004). However, illumination may change the species distribution and reactivity of the chlorine disinfectant (Oliver and Carey, 1977). Additionally, certain chlorination disinfection by-products may undergo further reactions and generate more toxic by-products when illuminated. For example, the chlorination disinfection by-products of triclosan generate more toxic tetrachlorodibenzo-p-dioxin in photo-chemical reactions (Buth et al., 2009).

The widely used BPs have access to treatment systems for drinking water, reclaimed water and swimming pool water. During the chlorination process, the residues of BPs can produce transformation products (TPs) that may be more toxic than parent compounds (Li et al., 2015). Tens of new products formed and the genotoxicity significantly increased in BP-4 chlorination (Xiao et al., 2013). Bromoform formed during the chlorination process of BP-3 in a seawater swimming pool (Manasfi et al., 2015). Several other studies have demonstrated that BPs may be transformed by photo-irradiation, and TPs have multiple toxic effects, such as photocarcinogenesis by DNA damage (Knowland et al., 1993). Benzophenone can be converted into 3-hydoxy-BP and 4-hydroxy-BP, which have stronger estrogenic toxicity after exposure to sunlight (Hayashi et al., 2006). BP-1 exhibited photogenotoxicity and decreased cell viability by $80.06 \%, 60.98 \%$ and 56.24\% under sunlight, UVA and UVB, respectively (Amar et al., 2015). However, comprehensive research on toxicity changes of BPs in a photoinduction-chlorination combined process has been less studied and is needed.

Therefore, 11 BPs were selected as target compounds in this study. The transformation characteristics and acute toxicity variation before and after a photoinduction-chlorination process were investigated, and the effects of their molecular structures and operational conditions, including $\mathrm{pH}$ values and chlorine dosages, are discussed. The study provides first-hand data for evaluating the potential risk of BPs in the environment and will provide excellent references regarding how to perform photoinduction-chlorination disinfection processes in practice.

\section{Materials and methods}

\subsection{Reagents and chemicals}

The 11 benzophenone compounds (>97.5\% purity) listed in Table 1 were purchased from Sigma-Aldrich (St. Louis, MO,

Table 1 - The 11 benzophenones UV-filters.

\begin{tabular}{|c|c|c|c|c|}
\hline BPs & Compound & Formula & MW & CAS No. \\
\hline B1 & Benzophenone & $\mathrm{C}_{13} \mathrm{H}_{10} \mathrm{O}$ & 182.22 & $119-61-9$ \\
\hline B2 & 2-Hydroxyl benzophenone & $\mathrm{C}_{13} \mathrm{H}_{10} \mathrm{O}_{2}$ & 198.22 & $117-99-7$ \\
\hline B3 & 3-Hydroxyl benzophenone & $\mathrm{C}_{13} \mathrm{H}_{10} \mathrm{O}_{2}$ & 198.22 & $13,020-57-0$ \\
\hline B4 & 4-Hydroxyl benzophenone & $\mathrm{C}_{13} \mathrm{H}_{10} \mathrm{O}_{2}$ & 198.22 & $1137-42-4$ \\
\hline B5 & 2,2'-Dihydroxyl benzophenone & $\mathrm{C}_{13} \mathrm{H}_{10} \mathrm{O}_{3}$ & 214.22 & $835-11-0$ \\
\hline B6 & 4,4'-Dihydroxyl benzophenone & $\mathrm{C}_{13} \mathrm{H}_{10} \mathrm{O}_{3}$ & 214.22 & $611-99-4$ \\
\hline B7 & 2,4-Dihydroxyl benzophenone & $\mathrm{C}_{13} \mathrm{H}_{10} \mathrm{O}_{3}$ & 214.22 & $131-56-6$ \\
\hline B8 & 2,4,4'-Trihydroxyl benzophenone & $\mathrm{C}_{13} \mathrm{H}_{10} \mathrm{O}_{4}$ & 230.22 & $1470-79-7$ \\
\hline B9 & 2,3,4-Trihydroxyl benzophenone & $\mathrm{C}_{13} \mathrm{H}_{10} \mathrm{O}_{4}$ & 230.22 & $1143-72-2$ \\
\hline B10 & 2,2',4,4'-Tetrahydroxyl benzophenone & $\mathrm{C}_{13} \mathrm{H}_{10} \mathrm{O}_{5}$ & 246.22 & $131-55-5$ \\
\hline B11 & 2-Hydroxy-4-methoxy benzophenone-5-sulfonic acid & $\mathrm{C}_{14} \mathrm{H}_{12} \mathrm{O}_{6} \mathrm{~S}$ & 308.31 & $4065-45-6$ \\
\hline
\end{tabular}


USA). All compounds were directly used in both the photoinduction-chlorination experiments and acute toxicity tests without further purification. A NaClO (4\%) aqueous solution was purchased from Wako Co. (Tokyo, Japan). Salts of analytical grade used for preparing buffer solutions and culture medium of the photobacterium strain were obtained from Beijing Chemical Works (Beijing, China). Biological agents, such as yeast extract and tryptone, which were used for making the culture medium of the photobacterium, were of biological grade and obtained from AOBOX Co., Ltd. (Beijing, China). Ultrapure water, which was used as a solvent throughout the experiments, was generated from a Millipore purification system (Billerica, MA, USA).

\subsection{Photoinduction-chlorination experiment}

The photoinduction-chlorination experiment was conducted in a quartz photochemistry reactor with a magnetic stirring apparatus. The reactor had a water cooling system, which maintained the reaction temperature at $35^{\circ} \mathrm{C}$. A xenon lamp was used as a light source, where the wavelength of the light source was limited within 420 to $780 \mathrm{~nm}$, with a light irradiation intensity of $400 \mathrm{~mW} / \mathrm{cm}^{2}$. The experiment was conducted as an orthogonal experiment at three different $\mathrm{pH}$ values and $\mathrm{NaClO}$ dosages. The $\mathrm{pH}$ values were set as 5, 7.5 and 10 , and the FAC dosages were set as 1-, 5-, and 10-equivalent to molar concentration of BPs, respectively. The reaction was initiated by adding the NaClO solution to $50 \mathrm{~mL}$ of the buffer solution, which included the appropriate concentration of BPs with illumination simultaneously; this solution was quenched by $\mathrm{Na}_{2} \mathrm{SO}_{3}$ solution immediately after $1 \mathrm{hr}$. The molar equivalent of $\mathrm{Na}_{2} \mathrm{SO}_{3}$ was 1.2 times that of the free available chlorine (FAC). The buffer solutions without BPs underwent a similar treatment and were used as the blank control. All experiments were performed in triplicate. All samples were stored in a refrigerator $\left(4^{\circ} \mathrm{C}\right)$ for the toxicity test.

\subsection{Photobacterium acute toxicity test}

The photobacterium acute toxicity bioassay was used to screen the relative toxicity variation of BPs before and after the photoinduction-chlorination process because this bioassay is a quick, sensitive, convenient and is the standardized toxicity test method. The test bacteria P. phosphoreum T3 freeze-dried powder was provided by the Institute of Soil Science, Chinese Academy of Sciences (Nanjing, China). The acute toxicity test with $P$. phosphoreum followed the recommendation of ISO 11348-3 (ISO, 2007) with some modifications. To guarantee the quality of the toxicity test, a $\mathrm{Zn}^{2+}$ solution, $3 \%$ $\mathrm{NaCl}$ solution and diluted buffer solution were set as the positive control, negative control and solvent control, respectively. $\mathrm{Zn}^{2+}$ was selected as the positive reference because it exhibits sensitivity, has a stable toxicity effect in the photobacterium toxicity test and is less harmful to environmental organisms and experiment operators. In each test, the dose-response curve of the positive control $\left(\mathrm{Zn}^{2+}\right)$ was prepared. For each BP parent compound, a series of solutions of gradient concentrations were prepared for the toxicity test, and the median effect concentration of 50\% (EC50) was obtained from the dose-response curve. The initial concentration of each BP in the photoinduction- chlorination process was set at EC50, and the acute toxicity of the samples before and after treatment was measured simultaneously. The exposure period was $15 \mathrm{~min}$, and the bioluminescence intensity of the photobacterium was measured immediately (Liu et al., 2014).

The toxicity effect of each sample was expressed as the relative bioluminescence inhibition ratio (IR, \%) to photobacterium:

$\mathrm{IR}=100 \%-\mathrm{L}_{\mathrm{SAMPLE}} / \mathrm{L}_{\mathrm{NC}} \times 100 \%$

where, $L_{\text {SAMPLE }}$ and $L_{\mathrm{NC}}$ represent the bioluminescence intensity of the sample and negative control, respectively. The IR of each sample was converted into the equivalent concentration of positive reference $\mathrm{Zn}^{2+}$ according to the doseresponse curve of $\mathrm{Zn}^{2+}$. Therefore, the toxicity of each sample can be expressed as the toxicity equivalent concentration of $\mathrm{Zn}^{2+}$, abbreviated as TEQ. Furthermore, to conveniently describe the acute toxicity variation and to clearly evaluate the potential risk of BPs during the photoinduction-chlorination process, the index of acute toxicity formation potential (ATFP) was proposed accordingly (Liu et al., 2014):

$\mathrm{ATFP}=\mathrm{TEQ}_{\mathrm{after}} / \mathrm{TEQ}_{\text {before }}$

where, $\mathrm{TEQ}_{\text {before }}$ and $\mathrm{TEQ} \mathrm{Qfter}_{\text {fefer }}$ to the toxicity equivalents before and after the photoinduction-chlorination process, respectively. Using the above formula, the relative variation of acute toxicity for BPs during photoinduction-chlorination treatment was obtained. An ATFP $>1$ indicates an increase in acute toxicity after treatment, whereas an ATFP $<1$ indicates a decrease in acute toxicity after treatment. The higher the ATFP value is, the greater the increase is in the acute toxicity after the photoinduction-chlorination process. Significant test on the TEQ for each sample before and after treatment was carried out by t-test. Additionally, the error bar of ATFP for each sample was shown in the figures, the toxicity difference among the samples will easily be understood.

\section{Results and discussion}

The acute toxicity variation of the 11 BPs during photoinductionchlorination disinfection treatment is evaluated by the ATFP values. All of the 11 BPs have the same benzophenone skeleton, and thus, the variation in acute toxicity after photoinduction-chlorination treatment can be attributed to the difference of the substituents and operational conditions. The effects of the substituent number, positions, types, $\mathrm{pH}$ values and chlorine dosages on the toxicity variation will be discussed.

\subsection{Effects of the hydroxyl group number}

Hydroxyl groups are the primary substituents in the 11 BPs molecules. The toxicity data showed that the ATFP values decreased as the number of hydroxyl groups on the benzene rings increased, i.e., BPs with fewer hydroxyl groups had a more significant toxicity elevation after the photoinductionchlorination treatment. Using B1, B2, B7 and B9 as examples, 
the number of hydroxyl groups in these samples are 0, 1, 2 and 3 , respectively. The acute toxicity increased (ATFP $>1$ ) for B1 after the photoinduction-chlorination treatment, whereas the toxicity decreased (ATFP < 1) for B2, B7 and B9 after treatment. The degree of toxicity decreased in the following order: B2 $<$ B7 < B9. As shown in Fig. 1, the ATFPs of 4 BPs exhibited a similar tendency under different $\mathrm{pH}$ conditions. B1 without a hydroxyl group exhibited a relatively low reaction activity with FAC, where the transformation ratio was less than $5 \%$. The ATFP of B1 is likely greater than 1 because of the formation of more toxic products than B1. Hayashi et al. (2006) reported that $\mathrm{B} 1$ can be converted to 3-hydroxy-BP (B3) and 4-hydroxy-BP (B4) after exposure to UV or sunlight. The toxicity test results of parent BPs demonstrated that B3 and B4 had higher acute toxicities than that of B1 (Liu et al., 2015). B2 has one hydroxyl group at the 2-position, whereas the hydroxyl group easily forms an intra-molecular hydrogen bond with the nearby carbonyl group, and thus, its reactivity would decrease. The ATFP of B2 was less than 1 after treatment due to less formation of less toxic products than that of the parent. B7 and B9 contain two and three hydroxyl groups, respectively, which have a high reaction activity and transformation ratios (Liu et al., 2014). On the one hand, it has been demonstrated that the acute toxicity of parent BPs increased as the hydroxyl group number increased in $P$. phosphoreum, Daphnia magna and Dugesia japonica (Liu et al., 2015; Li, 2012). On the other hand, a study by Liu et al. (2014) showed that B7 and B9 could generate several low-toxicity products, such as phenyl benzoate esters, during the chlorination process. Additionally, B7 could be $100 \%$ removed after $24 \mathrm{hr}$ of UV irradiation, where the products exert less estrogenic effects than that of the parent compound (Gago-Ferrero et al., 2012).

\subsection{Effects of the hydroxyl group position}

The position of substituents on benzene rings can also affect the toxicity variation. For example, B2, B3 and B4 contain one hydroxyl group on the 2-, 3- and 4-position, respectively, where the ATFPs of B3 and B4 were greater than 1, whereas the ATFP of B2 was lower than 1 (Fig. 2a). Similarly, B5 and B6 contain two hydroxyl groups, where B6 has two hydroxyl groups on the $4,4^{\prime}$-positions and had a higher ATFP than that of B5, which has two hydroxyl groups on the $2,2^{\prime}$-positions
(Fig. 2b). The hydroxyl group on the 4-position promotes the chlorination electrophilic substitution that occurred on the 3and 5-position, whereas the hydroxyl group on the 2-position formed an intramolecular hydrogen bond with the nearby carbonyl group and decrease the reactivity. Consequently, BPs containing 4-position hydroxyl may generate more toxic chlorinated products. The result was similar to the endocrine disrupting activity of the BPs in the following order: $2-<3-<(\approx)$ 4-position hydroxyl group. The 3-position hydroxyl group of BPs had a similar effect with that of the 4-position hydroxyl group because the 3-hydroxyl group binds the receptor at essentially the same degree as the 4-position group (Kawamura et al., 2003).

There is another case in which several BPs contain the same number of hydroxyl groups, where the distribution of hydroxyl groups on the benzene ring differ. For certain BPs, all of the hydroxyl groups are located on one benzene ring, whereas for others, the hydroxyl groups are located on two benzene rings. It can be concluded from Fig. $2 c$ and $d$ that the BPs with hydroxyl groups on two benzene rings clearly increased in acute toxicity after the photoinduction-chlorination treatment. As shown in Fig. 2c, B5 and B6 with two hydroxyl groups on two benzene rings (2,2'-, and 4,4'-positions) had higher ATFPs than that of B7 with two hydroxyl groups on one benzene ring (2,4-positions). Similarly, Fig. 2d shows that B8 with three hydroxyl groups on two benzene rings (2,2',4-positions) had a higher ATFP than that of B9 with three hydroxyl groups on one benzene ring (2,3,4-positions). This result is in accordance with that of BP chlorination treatment (Liu et al., 2014). A possible reason may be due to the stronger migratory aptitude (relative ability of a migrating group to migrate in a rearrangement reaction) of hydroxyl groups when they are located on one benzene ring than those located on two benzene rings. It has been demonstrated that the aromatic rings with more hydroxyl groups are readily cleavage in aqueous chlorination (Lindstrom and Nordin, 1976).

\subsection{Effects of several other substituents}

In addition to the hydroxyl group, there are several other substituents, such as the methoxyl group and sulfonic acid group, in the B11 molecule. The impact of these substituents on the acute toxicity variation in photoinduction-chlorination treatment was also investigated. To compare with B2 (2-hydroxyl-BP), a methoxyl group and a sulfonic acid group
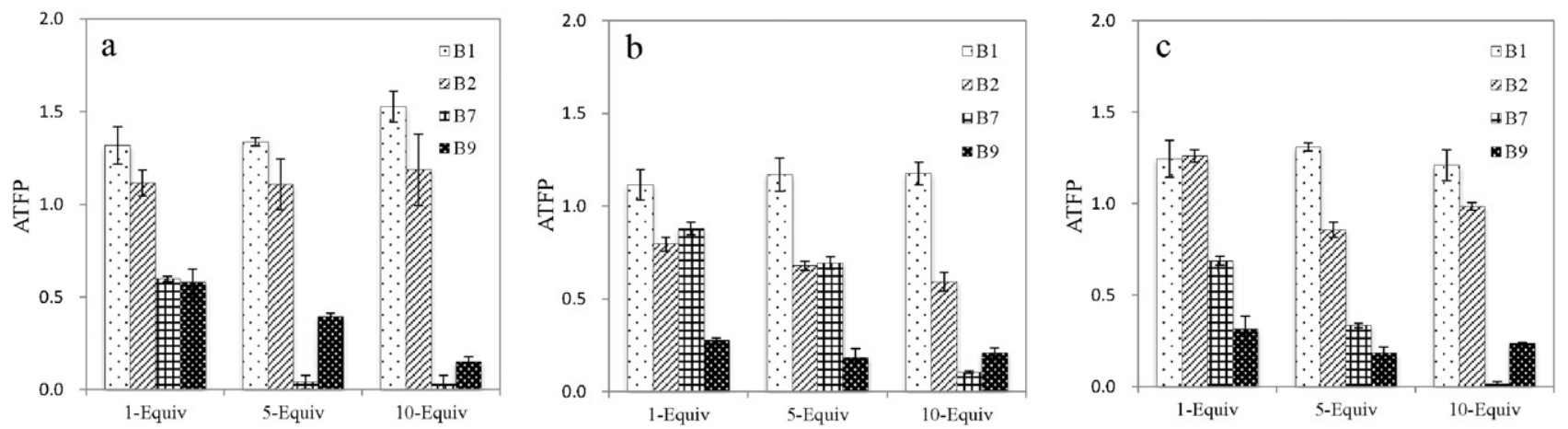

Fig. 1 - The relationship between hydroxyl number and acute toxicity formation potential (ATFP) of BPs in photo induction-chlorination treatment. Experimental conditions: (a) pH 5, (b) pH 7.5, (c) pH 10. Equiv: equivalent. BPs: benzophenones. 

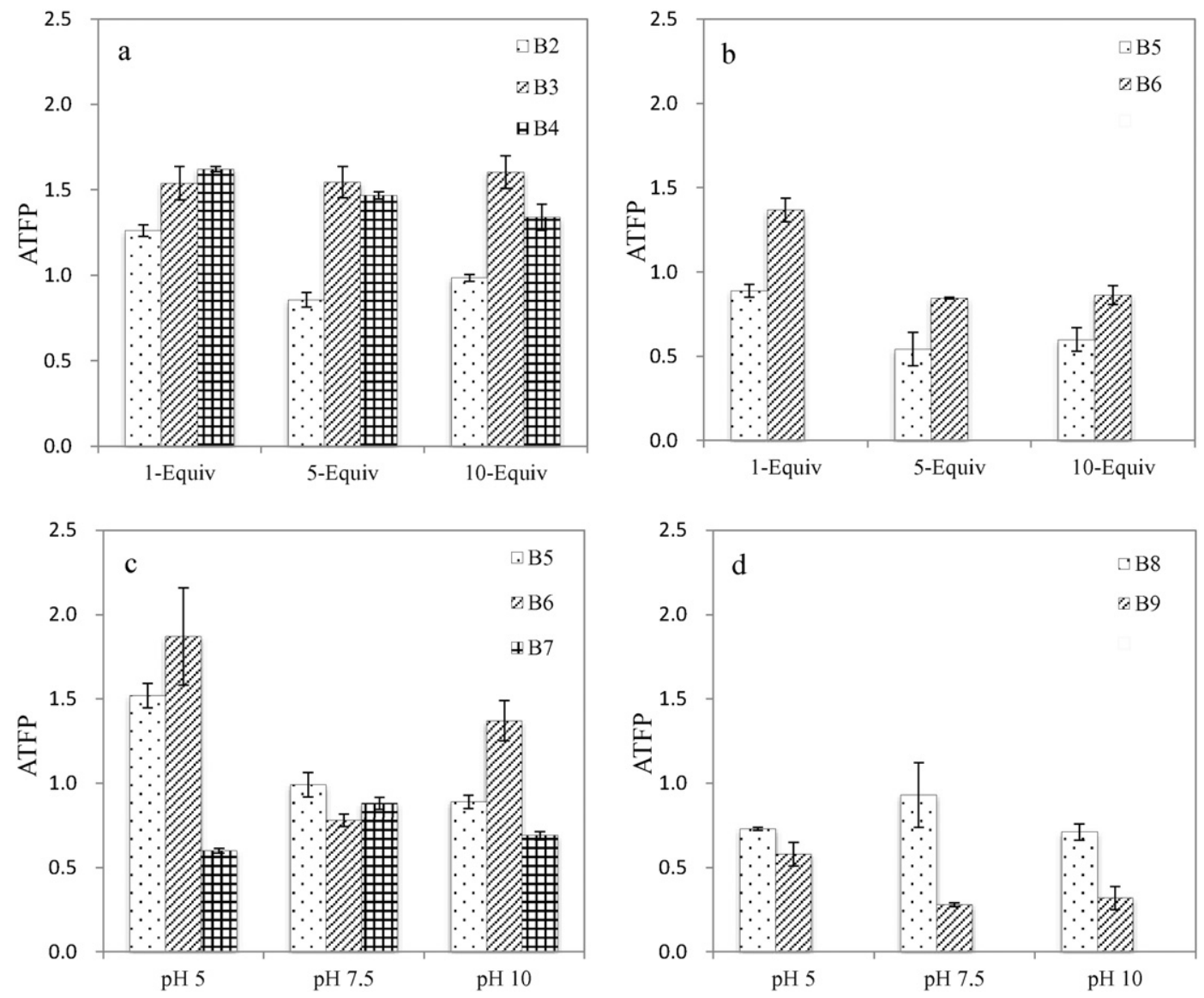

Fig. 2 - The acute toxicity formation potential (ATFP) of BPs with different hydroxyl group position in photo induction-chlorination treatment. Experimental conditions: (a) pH 10; (b) pH 10; (c) $[\mathrm{FAC}]_{0}:[\mathrm{BPs}]_{0}=1 ;$ (d) $[\mathrm{FAC}]_{0}:[\mathrm{BPs}]_{0}=1$.

were introduced in B11 (2-hydroxyl-4-methoxyl-5-sulfonic acid-BP). As shown in Fig. 3, the introduction of the methoxyl group and sulfonic acid group enhanced the ATFP value after photoinduction-chlorination treatment. This pattern remained at three different $\mathrm{pH}$ values. B11 had a much higher ATFP than that of B2 under the acid condition, whereas the ATFP of B11 was slightly greater than that of B2 under the alkaline condition. This result may be attributed to the different reaction types of B11 at different $\mathrm{pH}$ values $(\mathrm{pH}$ of 5, 7.5 and 10). B11 exhibited a high ATFP in the chlorination process due to the low toxicity of the BP parents and the formation of highly toxic products. B11 had a low toxicity effect because the sulfonic acid group increases the hydrophilicity, and the methoxyl group enhances the obstruction into cells (Liu et al., 2015; Zhao et al., 2013). For the chlorination process, B11 underwent desulfonation and generated a variety of toxic products with nearly a $100 \%$ transformation ratio (Xiao et al., 2013). For the photochemical process, B11 underwent indirect photolysis with $\mathrm{OH}$. and generated highly toxic products in the acid photoirradiation system, whereas it underwent direct
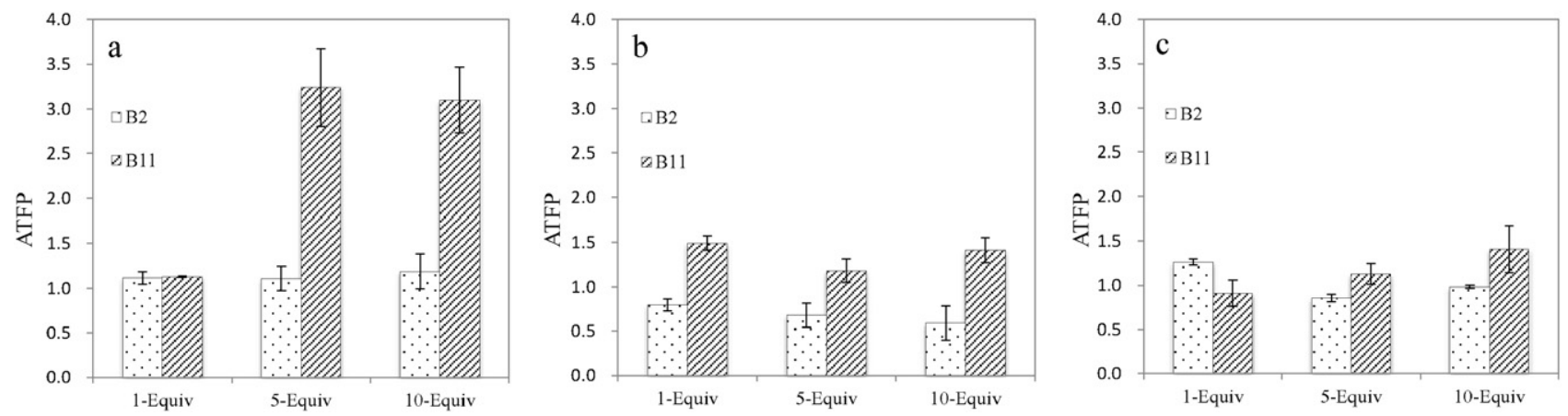

Fig. 3 - The correlation of acute toxicity formation potential (ATFP) with other substituents for BPs in photo-chlorination treatment. Experimental conditions: (a) pH 5, (b) pH 7.5, (c) pH 10. 


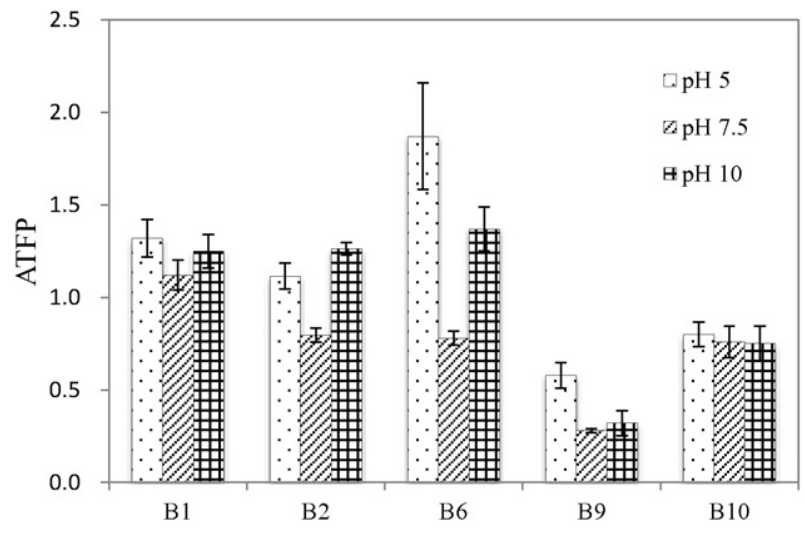

Fig. 4 - The pH-dependent variation of acute toxicity formation potential (ATFP) for BPs during photo induction-chlorination treatment. Experimental conditions: $[\mathrm{FAC}]_{0}:[\mathrm{BPs}]_{0}=1$.

photolysis and formed low-toxicity products in the alkaline system (Elisa et al., 2013).

\subsection{Effects of the $p H$ values}

In addition to the molecular structures, the operational conditions also impact the acute toxicity variation of BPs in photoinduction-chlorination treatment. The ATFPs for most of the selected BPs increased (ATFP $>1$ ) under acid and alkaline conditions but decreased (ATFP $<1$ ) under the neutral condition. As shown in Fig. 4, for each BP, the ATFP values increased in the following order: neutral < alkaline < acid condition. For some BPs, after photoinduction-chlorination treatment, the acute toxicity obviously increased under an acid condition, slightly increased under an alkaline condition and slightly decreased under a neutral condition. It is well known that $\mathrm{pH}$ values have great influence on the species and distribution of both the FAC and substrates (i.e., BPs). Under an acid condition, more reactions readily occur, and more toxic products are generated in BP-4 (denoted as B11 in this study) chlorination (Xiao et al., 2013). Oliver and Carey (1977) explored the photo-chlorination of ethanol, $n$-butanol and benzonic acid under 350-nm ultraviolet irradiation in different $\mathrm{pH}$ systems, where the results showed that UV irradiation affected the FAC reactivity. At pH 4 and $\mathrm{pH} 7,100 \%$ and 70\% FAC exists as HClO, which would generate highly reactive $\mathrm{OH}$. and $\mathrm{Cl}$. radicals after UV irradiation. At $\mathrm{pH} 10, \mathrm{ClO}^{-}$is the main species of FAC, which would generate $\mathrm{Cl}^{-}$and $\mathrm{O}$ with low reactivity. When irradiated at $254 \mathrm{~nm}$ under $\mathrm{pH} 10$, FAC would generate $\mathrm{OH}$. instead. Moreover, Guo et al. (2016) demonstrated that lower pH values accelerated the degradation rate and promoted the mineralization of chlortoluron in a UV/chlorination process. In this study, under an acid condition, BPs may have been attacked by abundant $\mathrm{OH}$. and $\mathrm{Cl}$., and highly toxic products, such as hydroxylated and chlorinated products, were generated. While under alkaline and neutral conditions, the reaction of BPs with FAC was inadequate because less $\mathrm{OH}$. exists.

\subsection{Effects of chlorine dosages}

Chlorine dosage is another impact factor on the acute toxicity variation of BPs after photoinduction-chlorination treatment. An overall tendency was that as the chlorine dosage increased, the ATFPs of BPs increased and then decreased. B1, B2 and B3 in Fig. 5a contain 0 or 1 hydroxyl group, and their ATFPs increased as the chlorine dosage increased. B5 and B6 in Fig. 5b contain 2 hydroxyl groups, and as the chlorine dosage increased from 1- to 5-equiv., their ATFPs decreased slightly, whereas when the chlorine dosage increased to 10-equiv., their ATFPs decreased sharply. B8, B9 and B10 in Fig. 5c contain 3 or 4 hydroxyl groups, and their ATFPs decreased significantly as the chlorine dosage increased. As reported by Xiao et al. (2013), the chlorine dosages influence the reaction extent of BPs with FAC. During the chlorination treatment of BPs, 4 types of products were formed in sequence: chlorination products, oxidation products (e.g., esters), hydrolysis products (e.g., chlorinated phenols, benzoic acids) and benzene ring broken products (e.g., chlorinated methanes, chloroacetate acids). For BPs with less hydroxyl groups (from B1 to B3), the transformation ratio did not increase significantly as the FAC dosage increased (Liu et al., 2014); thus, their ATFPs only slightly increased due to the increased formation of toxic chlorinated products. B5 and B6 contain two hydroxyl groups, and their reaction activity was greater than that of B1 and B2. When the FAC dose was 1- and 5-equiv., they could not be transformed completely; when the dose became as high as 10-equiv., the transformation continued. Correspondingly, their ATFPs slightly decreased at 1- and 5-equiv. FAC but sharply decreased at 10 -equiv. FAC due to the formation of
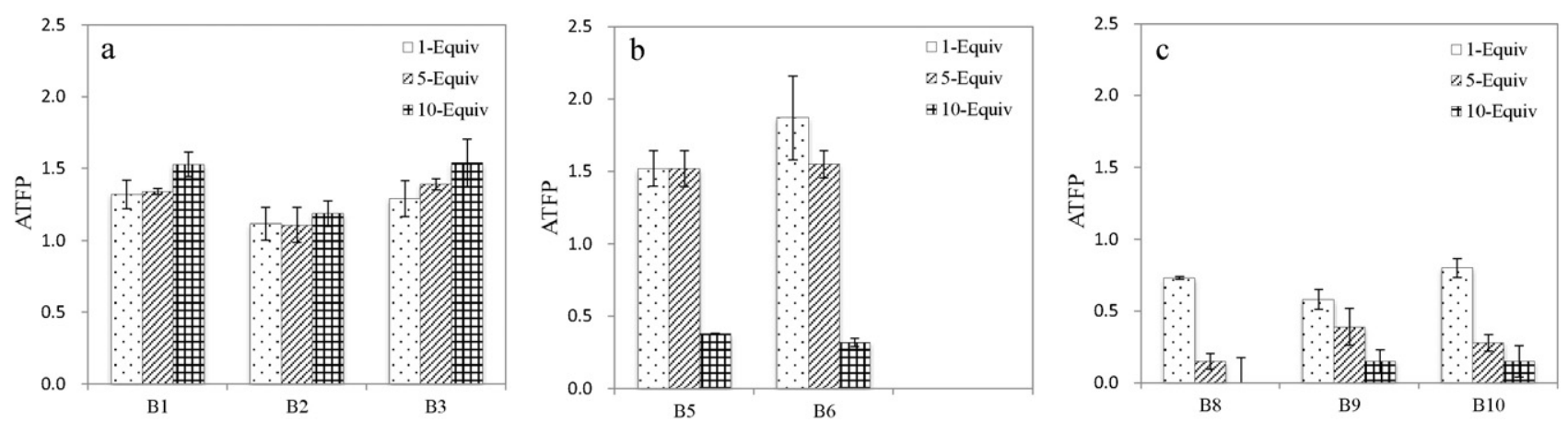

Fig. 5 - The FAC dose-dependent variation of acute toxicity formation potential (ATFP) values for BPs during photo induction-chlorination treatment. Experimental conditions: pH 5. FAC: free available chlorine. 
low-toxicity small molecular products (Xiao et al., 2013). Similarly, B8, B9 and B10 with three or four hydroxyl groups have a high reactivity and will have already thoroughly reacted with FAC at low FAC dosage. With increasing chlorine dosages, the ATFPs decreased due to increasingly smaller molecular products by decomposing parents and intermediate products. A study of Guo et al. (2016) proved that a higher chlorine dose could increase the degradation rate and promote the mineralization of chlortoluron during a UV/chlorine process. Wang et al. (2016) also found that in a UV/chlorination process, a higher chlorine dosage can easily enhance the iohexol degradation.

\section{Conclusions}

The photobacterium acute toxicity test was used to measure the toxicity variation of 11 BPs before and after photoinduction-chlorination disinfection treatment. The ATFP index was used to conveniently evaluate the acute toxicity variation of BPs after treatment. It can be concluded that the substituents types, hydroxyl group number and position, $\mathrm{pH}$ values and chlorine dosages had significant impacts on the ATFPs of BPs after photoinduction-chlorination treatment. The ATFPs decreased as the hydroxyl group number increased. The ATFPs are higher when the hydroxyl groups are located at the 3- or 4-position and distributed on two benzene rings. The introduction of the methoxyl group and sulfonic acid group elevates the ATFP values. The ATFPs are highest in an acid condition. The ATFPs increase and then decrease as the chlorine dosage increases. With the structure-dependent evaluation, the potential environmental fate of BPs was revealed and possible risks of chemical compounds with similar structures could be predicted. The operational condition-dependent results provide references to optimize parameters in water treatment practices. More importantly, BPs, as ultraviolet stabilizers and absorbers, were found to decompose and increase in toxicity when illuminated in several cases. However, the joint mechanism of photoinduction-chlorination disinfection treatment on $\mathrm{BPs}$ is unclear and requires further exploration.

\section{Acknowledgments}

This work was supported by the National Natural Science Foundation of China (Nos. 21577154, 21377143), and the Strategic Priority Research Program of the Chinese Academy of Sciences (No. XDB14040201).

\section{R E F E R E N C E S}

Almeida, C., Stepkowska, A., Alegre, A., Nogueira, J.M.F., 2013. Determination of trace levels of benzophenone-type ultra-violet filters in real matrices by bar adsorptive micro-extraction using selective sorbent phases. J. Chromatogr. A 1311, 1-10.

Amar, S.K., Goyal, S., Dubey, D., Srivastav, A.K., Chopra, D., Singh, J., et al., 2015. Benzophenone 1 induced photogenotoxicity and apoptosis via release of cytochrome c and Smac/DIABLO at environmental UV radiation. Toxicol. Lett. 239 (3), 182-193.
Asimakopoulos, A.G., Thomaidis, N.S., Kannan, K., 2014a. Widespread occurrence of bisphenol A diglycidyl ethers, p-hydroxybenzoic acid esters (parabens), benzophenone type-UV filters, triclosan, and triclocarban in human urine from Athens, Greece. Sci. Total Environ. 470-471, 1243-1249.

Asimakopoulos, A.G., Wang, L., Thomaidis, N.S., Kannana, K., 2014b. A multi-class bioanalytical methodology for the determination of bisphenol A diglycidyl ethers, p-hydroxybenzoic acid esters, benzophenone-type ultraviolet filters, triclosan, and triclocarban in human urine by liquid chromatography-tandem mass spectrometry. J. Chromatogr. A 1324, 141-148.

Buth, J.M., Grandbios, M., Vikesland, P.J., McNeill, K., Arnold, W.A., 2009. Aquatic photochemistry of chlorinated triclosan derivatives potential source of polychlorodibenzo-p-dioxins. Environ. Toxicol. Chem. 28 (12), 2555-2563.

Careghini, A., Mastorgio, A.F., Saponaro, S., Sezenna, E., 2015. Bisphenol a, nonylphenols, benzophenones, and benzotriazoles in soils, groundwater, surface water, sediments, and food: a review. Environ. Sci. Pollut. Res. 22 (8), 5711-5741.

EEC (European Economic Community), 1983. Council Directive 83/574/EEC of 26 October 1983 Amending for the Third Time Directive 76/768/EEC on the Approximation of the Laws of the Member States Relating to Cosmetic Products 332 pp. 38-42.

Elisa, D.L., Marco, M., Mohamed, S., Alessandro, M., Claudio, M., Gilles, M., et al., 2013. Photochemical processes involving the UV absorber benzophenone-4 (2-hydroxy-4methoxybenzophenone-5-sulphonic acid) in aqueous solution: reaction pathways and implications for surface waters. Water Res. 47 (15), 5943-5953.

Fent, K., Zenker, A., Rapp, M., 2010. Widespread occurrence of estrogenic UV-filters in aquatic ecosystems in Switzerland. Environ. Pollut. 158 (5), 1817-1824.

Gago-Ferrero, P., Badia-Fabregat, M., Olivares, A., Piña, B., Blánquez, P., Vicent, T., et al., 2012. Evaluation of fungal- and photo-degradation as potential treatments for the removal of sunscreens BP3 and BP1. Sci. Total Environ. 427-428, 355-363.

Gago-Ferrero, P., Mastroianni, N., Diaz-Cruz, M.S., Barcelo, D., 2013. Fully automated determination of nine ultraviolet filters and transformation products in natural waters and wastewaters by on-line solid phase extraction-liquid chromatography-tandem mass spectrometry. J. Chromatogr. A 1294, 106-116.

Guo, Z.B., Lin, Y.L., Xu, B., Huang, H., Zhang, T.Y., Tian, F.X., et al., 2016. Degradation of chlortoluron during UV irradiation and $\mathrm{UV} /$ chlorine processes and formation of disinfection by-products in sequential chlorination. Chem. Eng. J. 283, 412-419.

Hayashi, T., Okamoto, Y., Ueda, K., Kojima, N., 2006. Formation of estrogenic products from benzophenone after exposure to sunlight. Toxicol. Lett. 167 (1), 1-7.

ISO (International Organization for Standardization), 2007. Water Quality — Determination of the Inhibitory Effect of Water Samples on the Light Emission of Vibrio fischeri (Luminescent Bacteria Test) - Part 3: Method Using Freeze-Dried Bacteria (ISO11348-3). Geneva, Switzerland.

Janjua, N.R., Kongsho, B., Andersson, A.M., Wulf, H.C., 2008. Sunscreens in human plasma and urine after repeated whole-body topical application. J. Eur. Acad. Dermatol. Venereol. 22 (4), 456-461.

Jeon, H.K., Chung, Y., Ryu, J.C., 2006. Simultaneous determination of benzophenone-type UV filters in water and soil by gas chromatography-mass spectrometry. J. Chromatogr. A 1131 (1-2), 192-202.

Jeon, H.K., Sarma, S.N., Kim, Y.J., Ryu, J.C., 2007. Forward gene mutation assay of seven benzophenone-type UV filters using L5178Y mouse lymphoma cell. Mol. Cell. Toxicol. 3 (1), 23-30.

Kawamura, Y., Ogawa, Y., Nishimura, T., Kikuchi, Y., Nishikawa, J., Nishihara, T., et al., 2003. Estrogenic activities of UV stabilizers used in food contact plastics and benzophenone derivatives 
tested by the yeast two-hybrid assay. J. Health Sci. 49 (3), 205-212.

Knowland, J., McKenzie, E.A., McHugh, P.J., Cridland, N.A., 1993. Sunlight-induced mutagenicity of a common sunscreen ingredient. FEBS Lett. 324 (3), 309-313.

Kunisue, T., Chen, Z., Louis, G.M.B., Sundaram, R., Hediger, M.L., Sun, L.P., Kannan, K., 2012. Urinary concentrations of benzophenone-type UV filters in U.S. women and their association with endometriosis. Environ. Sci. Technol. 46 (8), 4624-4632.

Li, M.H., 2012. Acute toxicity of benzophenone-type UV filters and paraben preservatives to freshwater planarian, Dugesia japonica. Toxicol. Environ. Chem. 94 (3), 566-573.

Li, J., Ma, L.Y., Xu, L., Shi, Z.G., 2015. A novel two-dimensional liquid-chromatography method for online prediction of the toxicity of transformation products of benzophenones after water chlorination. Anal. Bioanal. Chem. 407 (20), 6137-6148.

Lindstrom, K., Nordin, J., 1976. Gas chromatography-mass spectrometry of chlorophenols in spent bleach liquours. J. Chromatogr. A 128, 13-26.

Liu, W.J., 2004. Forecast on advanced water disinfection. Water Wastewater Eng. 30 (1), 2-5.

Liu, Q., Chen, Z.B., Wei, D.B., Du, Y.G., 2014. Acute toxicity formation potential of benzophenone-type UV filters in chlorination disinfection process. J. Environ. Sci. 26 (2), 440-447.

Liu, H., Sun, P., Liu, H.X., Yang, S.G., Wang, L.S., Wang, Z.Y., 2015. Acute toxicity of benzophenone-type UV filters for Photobacterium phosphoreum and Daphnia magna: QSAR analysis, interspecies relationship and integrated assessment. Chemosphere 135, 182-188.

Louis, G.M.B., Kannan, K., Sapra, K.J., Maisoj, J., Sundaram, R., 2014. Urinary concentrations of benzophenone-type ultraviolet radiation filters and couples' fecundity. Am. J. Epidemiol. 180 (12), 1168-1175.

Manasfi, T., Storck, V., Ravier, S., Demelas, C., Coulomb, B., Boudenne, J.L., 2015. Degradation products of benzophenone-3 in chlorinated seawater swimming pools. Environ. Sci. Technol. 49 (15), 9308-9316.

MHLW (Ministry of Health and Welfare), 2000. Japanese Standards of Cosmetic Ingredients. Ministry of Health and Welfare Notification. No. 331, Japan.

Nancy, B., Sara, Z., Karl, F., 2012. Effects of the UV filter benzophenone-3 (oxybenzone) at low concentrations in zebrafish (Danio rerio). Toxicol. Appl. Pharmacol. 263 (2), 184-194.

NIES (National Institute for Environmental Studies), 1998. Strategic Programs on Environmental Endocrine Disruptors (SPEED'98), Analytical Methods for Endocrine Disruptors, Japan.

NTP (National Toxicology Program), 2006. Toxicology and carcinogenesis studies of benzophenone in F344/N rats and
B6C3F1 mice (feed studies). NTP TR 533. U.S. Department of Health and Human Services. NIH Publication No. 06-4469.

Oliver, B.G., Carey, J.H., 1977. Photochemical production of chlorinated organics in aqueous solutions containing chlorine. Environ. Sci. Technol. 11 (9), 893-895.

Pathak, M.A., 1987. Sunscreens and their use in the preventive treatment of sunlight-induced skin damage. J. Dermatol. Surg. Oncol. 13 (7), 739-752.

Richardson, S.D., Plewa, M.J., Wagner, E.D., Schoeny, R., DeMarini, D.M., 2007. Occurrence, genotoxicity, and carcinogenicity of regulated and emerging disinfection by-products in drinking water: a review and roadmap for research. Mutat. Res. Rev. Mutat. Res. 636 (1-3), 178-242.

Rodil, R., Quintana, J.B., Purification, L.M., Soledad, M.L., Dario, P.R., 2008. Multiclass determination of sunscreen chemicals in water samples by liquid chromatography-tandem mass spectrometry. Anal. Chem. 80 (4), 1307-1315.

Roelandts, R., Vanhee, J., Bonamie, A., Kerkhofs, L., Degreef, H., 1983. A survey of ultraviolet absorbers in commercially available sun products. Int. J. Dermatol. 22 (4), 247-255.

Schlumpf, M., Cotton, B., Conscience, M., Haller, V., Steinmann, B., Lichtensteiger, W., 2001. In vitro and in vivo estrogenicity of UV screens. Environ. Health Perspect. 109 (3), 239-244.

Wang, L., Asimakopoulos, A.G., Moon, H.B., Nakata, H., Kannan, H., 2013. Benzotriazole, benzothiazole, and benzophenone compounds in indoor dust from the United States and East Asian countries. Environ. Sci. Technol. 47 (9), 4752-4759.

Wang, Z., Lin, Y.L., Xu, B., Xia, S.J., Zhang, T.Y., Gao, N.Y., 2016. Degradation of iohexol by UV/chlorine process and formation of iodinated trihalomethanes during post-chlorination. Chem. Eng. J. 283, 1090-1096.

Weisbrod, C.J., Kunz, P.Y., Zenker, A.K., Fent, K., 2007. Effects of the UV filter benzophenone-2 on reproduction in fish. Toxicol. Appl. Pharmacol. 225 (3), 255-266.

Xiao, M., Wei, D.B., Yin, J.X., Wei, G.H., Du, Y.G., 2013. Transformation mechanism of benzophenone-4 in free chlorine promoted chlorination disinfection. Water Res. 47 (16), 6223-6233.

Yang, C., Wang, X.Y., Wang, D., Gu, N.J., 2011. Comparative analysis of reclaimed water disinfection by UV-chlorine combination and single chlorine process. Water Technol. 5 (1), 19-22.

Zhang, Z.F., Ren, N.Q., Li, Y.F., Kunisue, T., Gao, D.W., Kannan, K., 2011. Determination of benzotriazole and benzophenone UV filters in sediment and sewage sludge. Environ. Sci. Technol. 45 (9), 3909-3916.

Zhao, H.M., Wei, D.B., Li, M., Du, Y.G., 2013. Substituent contribution to the genotoxicity of benzophenone-type UV filters. Ecotoxicol. Environ. Saf. 95, 241-246. 